\title{
Atténuation acoustique basse fréquence dans l'eau de mer : étude des mécanismes de relaxation
}

\author{
P. Mallo, G. Waton et S. J. Candau \\ Laboratoire de Spectrométrie et d'Imagerie Ultrasonores (anciennement Laboratoire d'Acoustique Moléculaire), \\ Formation Associée au C.N.R.S., Université Louis Pasteur, 4, rue Blaise Pascal, 67070 Strasbourg Cedex, France
}

(Reçu le 4 octobre 1984, révisé le 11 janvier 1985, accepté le 4 février 1985)

\begin{abstract}
Résumé. - L'atténuation acoustique basse fréquence dans l'eau de mer a été calculée à partir d'un modèle de relaxation faisant intervenir différents constituants de l'eau de mer. Les prédictions théoriques sont comparées aux résultats obtenus à partir des expériences de propagation à longue portée dans les océans.
\end{abstract}

\begin{abstract}
The low frequency sound attenuation in sea-water is calculated from a relaxational model involving different components of sea-water. The theoretical predictions are compared to the results of long range propagation experiments performed in oceans.
\end{abstract}

\section{Introduction.}

La dissipation des ondes acoustiques joue un rôle considérable dans les expériences de communication sous-marine, car elle limite la portée des ondes acoustiques. Malgré le grand nombre d'expériences de propagation acoustique réalisées au cours des trente dernières années dans les océans et les grands lacs, la compréhension du mécanisme d'atténuation des ondes sonores de basse fréquence $(100-10000 \mathrm{~Hz})$ pose encore de nombreux problèmes.

Avant d'aborder l'étude de l'absorption acoustique basse fréquence, il est utile de rappeler les principaux résultats obtenus en laboratoire dans le domaine des plus hautes fréquences. En 1949, Léonard et al. [1] ont montré à partir d'études réalisées au moyen de la technique du résonateur acoustique que la principale contribution à l'atténuation pour des fréquences supérieures à $10 \mathrm{kHz}$ provenait de la présence du sulfate de magnésium dans l'eau de mer. Ce résultat a été confirmé par Kurtze et Tamm [2], Wilson et Léonard [3] et Tamm et al. [4, 5] et il est universellement reconnu maintenant que cette contribution provient d'un mécanisme de dissociation du $\mathrm{MgSO}_{4}$.

Dans le domaine des basses fréquences, l'analyse de Thorp [6, 7] en 1965 et les travaux de Leroy et Skretting [8] en 1971 mirent en évidence un phénomène de relaxation autour de $1 \mathrm{kHz}$, spécifique à l'eau de mer, sans toutefois en identifier la cause.

Yaeger et al. [9] en 1973 montrèrent, sans aucuné ambiguité, en utilisant la technique du saut de tempé- rature, que le processus relaxationnel observé était dû à la présence d'acide borique, en très faible quantité $(0,43 \mathrm{mM}$ en moyenne) dans l'eau de mer. Ces résultats furent confirmés ultérieurement par Simmons [10] au moyen de la même technique; de plus, cet auteur observa que le mécanisme de base faisait intervenir les ions bicarbonate et carbonate présents dans l'eau de mer.

En fait, la fréquence de relaxation associée à l'acide borique est très inférieure au domaine de fréquences accessibles avec la technique du résonateur. En effet, pour une fréquence de $1 \mathrm{kHz}$, la longueur d'onde acoustique est de $1,5 \mathrm{~m}$, ce qui rend impossible toute mesure directe sur de l'eau de mer en laboratoire. Les informations sur le mécanisme de relaxation associé à l'acide borique ne peuvent être obtenues qu'à partir de solutions au moins dix fois plus concentrées en $\mathrm{B}(\mathrm{OH})_{3}$ que l'eau de mer. Les travaux très complets de Mellen [11] ont été réalisés dans ces conditions. L'analyse de ses mesures apparaît très délicate car dans ce domaine de concentration, d'une part, il y a formation d'espèces chimiques qui ne sont pas présentes en milieu marin, d'autre part, de nouveaux processus de relaxation apparaissent. Cependant, cette étude a montré très nettement que le modèle simple de relaxation proposé initialement [9] ne pouvait rendre compte des résultats expérimentaux.

La technique du saut de température permet de travailler dans le domaine de $1 \mathrm{kHz}$ et par conséquent, d'étudier des solutions d'eau de mer en s'affranchissant des inconvénients de la technique acoustique. Les 
seules expériences réalisées à ce jour par cette méthode sont donc, les mesures de Yeager [9] qui ont permis l'identification de l'espèce relaxante et les travaux de Simmons [10]. Ces expériences, très limitées, ne sont pas suffisantes pour déterminer le mécanisme.

Récemment nous avons présenté une étude systématique par relaxation après saut de température des solutions d'acide borique, additionnées sélectivement des différents sels contenus dans l'eau de mer.

Ces travaux [12,13] ont permis d'établir le mécanisme réactionnel qui produit l'atténuation acoustique. Dans cet article, nous présentons le calcul, à partir $\mathrm{du}$ modèle réactionnel, des caractéristiques de la propagation sous-marine d'ondes acoustiques en relation avec les modifications océanographiques (température, pression, salinité, $\mathrm{pH}$ ). Une comparaison avec les résultats obtenus en mer est également effectuée.

\section{Mécanisme de relaxation chimique.}

Les résultats obtenus par relaxation après saut de température, nous ont conduits à proposer un modèle réactionnel fondé sur des transferts de protons entre deux couples acide-base : acide borique-borate et carbonate-bicarbonate [13]. Ce modèle peut être représenté par le schéma ci-dessous :

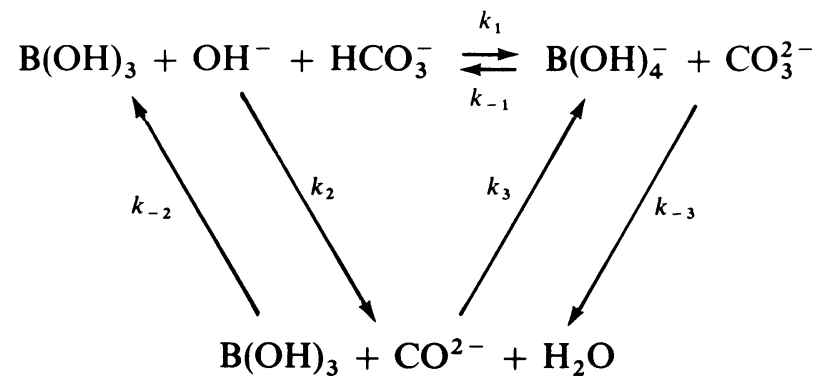

où $k_{i}$ et $k_{-i}(i=1,2,3)$ sont les constantes de vitesse directe et inverse.

L'équilibre $\mathrm{HCO}_{3}^{-}+\mathrm{OH}^{-} \rightleftarrows \mathrm{CO}_{3}^{2-}+\mathrm{H}_{2} \mathrm{O}$ étant rapide devant les deux autres, la résolution du système d'équations cinétiques pour un tel modèle conduit à deux fréquences de relaxation [13] : la première correspond à l'équilibre précédent, la deuxième plus basse est celle qui nous intéresse principalement dans le cadre de ce travail et s'écrit :

$$
\begin{aligned}
f_{\mathrm{r}} & =\frac{1}{2 \pi} \mid k_{1}\left(\left|\mathrm{~B}(\mathrm{OH})_{3}\right| \frac{1}{1+\alpha}+\left|\mathrm{OH}^{-}\right|\right)+\frac{k_{1}}{K_{1}}+ \\
& +k_{3}\left(\left|\mathrm{CO}_{3}^{2-}\right|+\frac{\alpha}{1+\alpha}\left|\mathrm{B}(\mathrm{OH})_{3}\right|\right)+ \\
& +\frac{k_{3}}{K_{3}}\left(\left|\mathrm{~B}(\mathrm{OH})_{4}^{-}\right| \frac{\alpha}{1+\cdot \alpha}+\left|\mathrm{HCO}_{3}^{-}\right|\right) \mid
\end{aligned}
$$

avec

$$
\alpha=\frac{K_{2}\left|\mathrm{HCO}_{3}^{-}\right|}{1+K_{2}\left|\mathrm{OH}^{-}\right|}
$$

$K_{1}, K_{2}, K_{3}$ les constantes d'équilibres thermodynamiques sont relevées dans la littérature [13].
Sur les figures 1 et 2 nous avons reporté les variations de $f_{\mathrm{r}}$ en fonction respectivement de la concentration en $\left(\mathrm{HCO}_{3}^{-}\right)$et du $\mathrm{pH}$ pour des solutions synthétiques. Nous avons également représenté les variations de $f_{\mathrm{r}}$, calculées par ajustement de l'équation (2). Le seul paramètre ajustable était $k_{3}, k_{1}$ ayant été déterminé indépendamment [12].

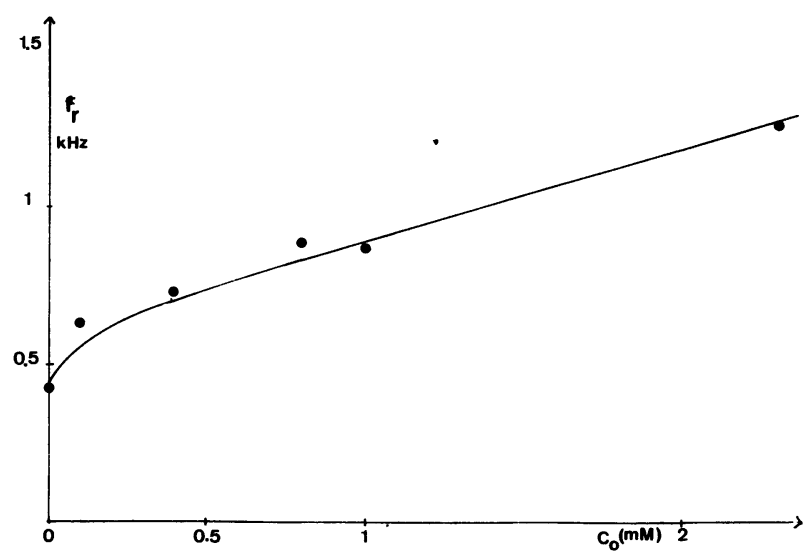

Fig. 1. - Variation de la fréquence de relaxation avec la concentration analytique $C_{0}$ en $\mathrm{HCO}_{3}^{-}$pour une solution; $0,43 \mathrm{mM} \mathrm{B}(\mathrm{OH})_{3}, \mathrm{pH}=9$. La courbe en trait plein représente le meilleur ajustement de l'équation (2) à nos données.

[Variation of the relaxation frequency with the concentration $C_{0}$ of $\mathrm{HCO}_{3}^{-}$for a $0.43 \mathrm{mM} \mathrm{B}(\mathrm{OH})_{3}$ solution at $\mathrm{pH}=9$. The full line represents the best fit of the equation (2) to the experimental data.]

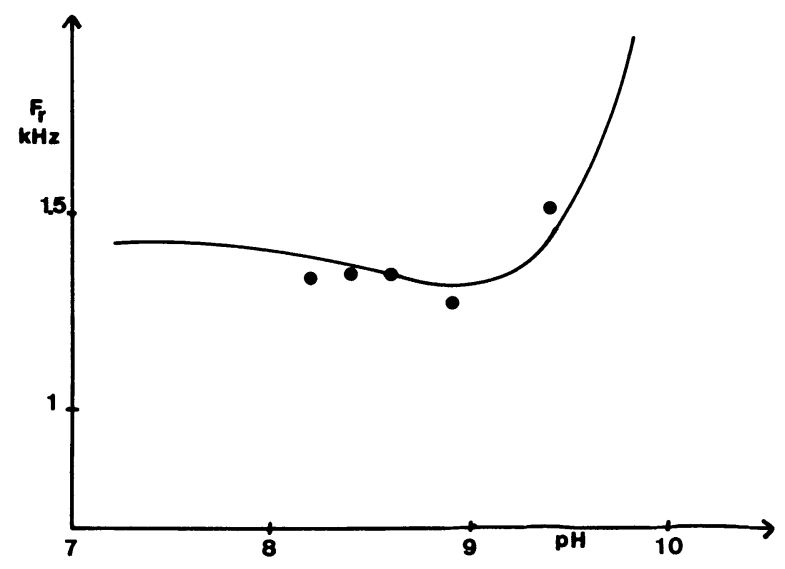

Fig. 2. - Variation de la fréquence de relaxation en fonction du $\mathrm{pH}$ pour une solution $0,43 \mathrm{mM} \mathrm{B}(\mathrm{OH})_{3}$ et $1 \mathrm{mM} \mathrm{HCO}_{3}^{-}$. La courbe en trait plein représente le meilleur ajustement de l'équation (2) à nos données.

[Variation of the relaxation frequency as a function of $\mathrm{pH}$ for a solution at $0.43 \mathrm{mM} \mathrm{B}(\mathrm{OH})_{3}$ and $1 \mathrm{mM} \mathrm{HCO}_{3}^{-}$. The full line represents the best fit of the equation (2) to the experimental data.]

\section{Atténuation acoustique.}

La présence d'un processus relaxationnel produit une atténuation acoustique supplémentaire présentant un maximum à la fréquence de relaxation [14]. 
Le maximum d'atténuation acoustique, pour le mécanisme modélisé par le schéma triangulaire précédent est donné par [15] :

$$
\begin{aligned}
& \alpha_{\max }=\left(\frac{\rho_{0} v_{\mathrm{s}}}{2 R T}\right)(\Delta V)^{2} \times \\
& \times\left(k_{1}\left|\mathrm{~B}(\mathrm{OH})_{3}\right|\left|\mathrm{OH}^{-}\right|+k_{3}\left|\mathrm{~B}(\mathrm{OH})_{3}\right|\left|\mathrm{CO}_{3}^{2-}\right|\right) .
\end{aligned}
$$

Dans cette relation, $\Delta V$ désigne le changement de volume global impliqué dans le mécanisme; $\rho_{0}$ la densité de l'eau, $v_{\mathrm{s}}$ la vitesse de propagation des ondes acoustiques, $R$ et $T$ la constante des gaz parfaits et la température absolue.

$\Delta V$ peut être calculé en écrivant :

$$
\Delta V \Delta \mathrm{B}(\mathrm{OH})_{4}^{-}=-\Delta V_{1} \Delta \mathrm{OH}^{-}-\Delta V_{3} \Delta \mathrm{CO}_{3}^{2-}
$$

où $\Delta V_{1}$ est le changement de volume molaire associé à l'équilibre

$$
\mathrm{B}(\mathrm{OH})_{3}+\mathrm{OH}^{-} \rightleftarrows \mathrm{B}(\mathrm{OH})_{4}^{-}
$$

et $\Delta V_{3}$ le changement de volume molaire associé à l'équilibre d'échange

$$
\mathrm{B}(\mathrm{OH})_{3}+\mathrm{HCO}_{3}^{-} \rightleftarrows \mathrm{CO}_{3}^{2-}+\mathrm{B}(\mathrm{OH})_{4}^{-}+\mathrm{H}_{2} \mathrm{O} .
$$

En écrivant les équations de conservation de la matière et en tenant compte des équilibres chimiques, on obtient l'expression de $\Delta V$ :

$$
\begin{aligned}
\Delta V=\Delta V_{1} & \frac{1+K_{2}\left|\mathrm{OH}^{-}\right|}{1+K_{2}\left(\left|\mathrm{OH}^{-}\right|+\left|\mathrm{HCO}_{3}^{-}\right|\right)}+ \\
& +\Delta V_{3} \frac{K_{2}\left|\mathrm{HCO}_{3}^{-}\right|}{1+K_{2}\left(\left|\mathrm{OH}^{-}\right|+\left|\mathrm{HCO}_{3}^{-}\right|\right)} .
\end{aligned}
$$

Le pH de la mer est voisin de 8,1 et $\left|\mathrm{HCO}_{3}^{-}\right| \simeq$ 2,3 mM, par ailleurs $K_{2} \simeq 10^{5}$, ce qui permet d'écrire :

$$
\Delta V \simeq \Delta V_{3} \text {. }
$$

La variation de volume lors du déplacement des équilibres est donc due principalement à la réaction d'échange :

$$
\mathrm{H}_{2} \mathrm{O}+\mathrm{B}(\mathrm{OH})_{3}+\mathrm{HCO}_{3}^{-} \rightleftarrows \mathrm{B}(\mathrm{OH})_{4}^{-}+\mathrm{CO}_{3}^{2}
$$

$\Delta V_{3}$ est donné par :

$$
\Delta V_{3}=V_{\mathrm{B}(\mathrm{OH})_{4}^{-}}+V_{\mathrm{HCO}_{3}^{-}}-\left(V_{\mathrm{B}_{(\mathrm{OH})_{3}}}+V_{\mathrm{CO}_{3}^{2}-}+V_{\mathrm{H}_{2} \mathrm{O}}\right) .
$$

Les valeurs des volumes molaires partiels, mesurées par Millero [16,17], conduisent à :

$$
\Delta V_{3}-11 \mathrm{ml} / \mathrm{mole} \text {. }
$$

Le maximum d'atténuation acoustique est alors donné par :

$\alpha_{\max }=$

$=\frac{\left(\Delta V_{3}\right)^{2} \rho_{0} v_{\mathrm{s}}\left|\mathrm{B}(\mathrm{OH})_{3}\right|\left(k_{1}\left|\mathrm{OH}^{-}\right|+k_{3}\left|\mathrm{CO}_{3}^{2-}\right|\right)}{2 R T}$.

A partir de la salinité de la mer on peut, en utilisant des formules empiriques [11], obtenir les concentrations en $\mathrm{B}(\mathrm{OH})_{3}$ et $\mathrm{CO}_{3}^{2-}$; à partir du $\mathrm{pH}$ on accède à la concentration en $\mathrm{OH}^{-}$, enfin $k_{1}$ et $k_{3}$ dépendent directement de la température.

En se plaçant dans les conditions océanographiques correspondant à l'Atlantique Nord, c'est-à-dire une salinité de $34 \mathrm{~g} / 1, T=4^{\circ} \mathrm{C}, \mathrm{pH}=8,1$, nous pouvons calculer la valeur $\alpha_{\max }$,

$$
\alpha_{\max }=3,3 \times 10^{-2} \mathrm{~dB} / \mathrm{km} .
$$

La valeur mesurée en océan est de l'ordre de $0,12 \mathrm{~dB} / \mathrm{km}$ [6]. Par conséquent, notre modèle prévoit une atténuation trop faible et ce résultat est systématique pour toutes les mers.

Les valeurs trop faibles des atténuations obtenues peuvent s'expliquer par l'insuffisance du modèle « triangulaire ». Un traitement rigoureux du système doit en effet tenir compte des réactions de formation des paires d'ions, mettant en jeu les ions $\mathrm{CO}_{3}^{2-}$ comme l'a précédemment signalé Mellen [11]

$$
\begin{aligned}
& \mathrm{Ca}^{2+}+\mathrm{CO}_{3}^{2-} \rightleftarrows \mathrm{CaCO}_{3}^{0} \\
& \mathrm{Mg}^{2+}+\mathrm{CO}_{3}^{2-} \rightleftarrows \mathrm{MgCO}_{3}^{0} .
\end{aligned}
$$

Ces réactions peuvent s'inclure dans notre modèle que l'on écrit alors :

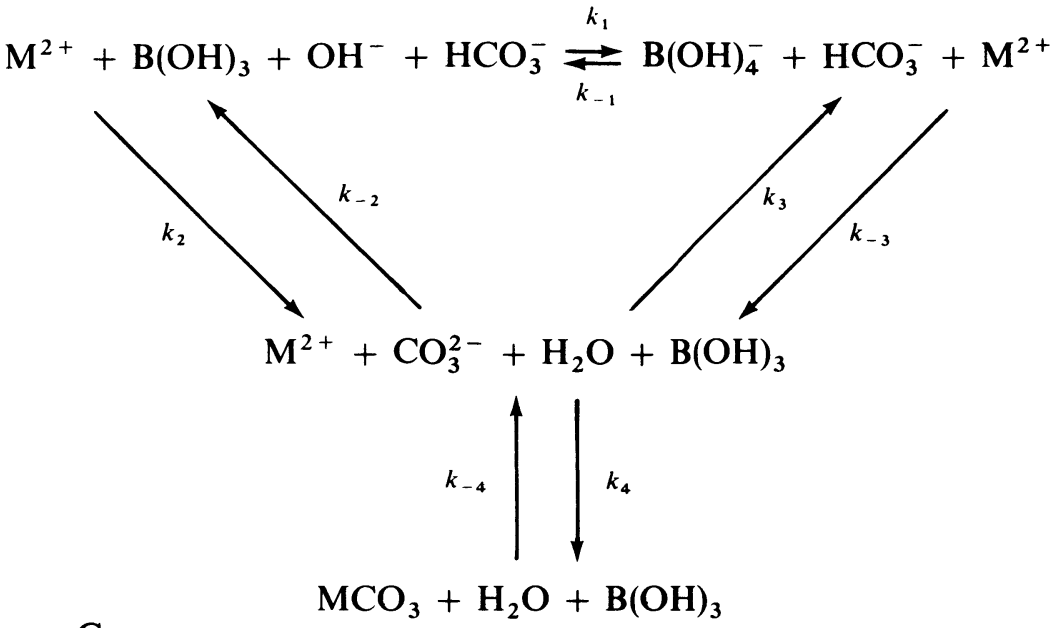

où $\mathrm{M}$ représente $\mathrm{Mg}$ ou $\mathrm{Ca}$. 
Le calcul de la fréquence de relaxation et de l'atténuation acoustique pour un tel schéma est très complexe. Néanmoins ce modèle peut être simplifié en remarquant que la concentration en $\mathrm{CO}_{3}^{2-}$ libre est en fait très faible dans la mer. Les ions $\mathrm{CO}_{3}^{2-}$ sont présents essentiellement sous la forme de paires d'ions $\mathrm{MgCO}_{3}, \mathrm{CaCO}_{3}^{0}$; il en résulte que la réaction d'échange acide borique-carbonate peut alors s'écrire :

$$
\mathrm{MCO}_{3}+\mathrm{B}(\mathrm{OH})_{3}+\mathrm{H}_{2} \mathrm{O} \underset{k-3}{\stackrel{k_{3}}{\rightleftarrows}} \mathrm{B}(\mathrm{OH})_{4}^{-}+\mathrm{HCO}_{3}^{-} .
$$

Nous avons montré précédemment que la variation de volume lors du déplacement des équilibres était essentiellement due à la réaction d'échange.

Par conséquent on peut écrire que la variation de volume global $\Delta V$ s'écrit :

$$
\Delta V=\Delta V_{3}-\Delta V_{4}
$$

$\Delta V_{4}$ est la variation de volume associée aux équilibres de formation des paires d'ions $\mathrm{MgCO}_{3}^{0}$ et $\mathrm{CaCO}_{3}^{0}$ [18]. $\mathrm{Mg}^{2+}+\mathrm{CO}_{3}^{2-} \rightleftarrows \mathrm{MgCO}_{3}^{0} \quad \Delta V_{41}=12,5 \mathrm{ml} / \mathrm{mol}$,

$$
\mathrm{Ca}^{2}+\mathrm{CO}_{3}^{2-} \rightleftarrows \mathrm{CaCO}_{3}^{0} \quad \Delta V_{42}=12,5 \mathrm{ml} / \mathrm{mol}
$$

$\Delta V_{41}=\Delta V_{42}$, ce qui simplifie nettement les calculs et on a alors $\Delta V_{4}=\Delta V_{41}=\Delta V_{42}$, ce qui conduit à :

$$
\Delta V=-24 \mathrm{ml} / \mathrm{mol} \text {. }
$$

Le maximum d'atténuation acoustique est alors donné par :

$$
\begin{aligned}
\alpha_{\max } & = \\
= & \frac{(\Delta V)^{2} \rho_{0} v_{\mathrm{s}}\left|\mathrm{B}(\mathrm{OH})_{3}\right|\left(k_{\mathrm{s}}|\mathrm{OH}|+k_{3}\left|\mathrm{CO}_{3}^{2-}\right|_{T}\right)}{2 R T}
\end{aligned}
$$

où $\left|\mathrm{CO}_{3}^{2-}\right|_{T}$ désigne la concentration totale (ions libres et paires d'ions) en $\mathrm{CO}_{3}^{2-}$. L'atténuation acoustique varie avec la fréquence selon l'équation :

$$
\alpha=\alpha_{\max }\left(\frac{f^{2}}{f^{2}+f_{\mathrm{r}}^{2}}\right) .
$$

La valeur de la fréquence de relaxation $f_{\mathrm{r}}$ est obtenue à partir du modèle proposé et se calcule par conséquent à partir de l'équation (2).

Le tableau I permet la comparaison des valeurs théoriques avec les résultats obtenus lors des campagnes de propagation acoustique longue portée réalisées en océan.

Les figures 3 à 5 représentent les variations calculées du coefficient d'atténuation acoustique en fonction de la fréquence. Pour obtenir ces variations, nous avons ajouté à la contribution basse fréquence calculée à partir de l'équation (18), le terme dû à la relaxation du
Tableau I. - Comparaison des fréquences de relaxation calculées par l'équation (2) et obtenues par propagation acoustique.

[Comparison between calculated and experimental values of the acoustical relaxation frequency in several seas.]

\begin{tabular}{|c|c|c|c|c|}
\cline { 2 - 5 } \multicolumn{1}{c|}{} & $\begin{array}{c}\text { Paci- } \\
\text { fique }\end{array}$ & $\begin{array}{c}\text { Atlan- } \\
\text { tique }\end{array}$ & $\begin{array}{c}\text { Méditer- } \\
\text { ranée }\end{array}$ & $\begin{array}{c}\text { Mer } \\
\text { Rouge }\end{array}$ \\
\hline$f_{\mathrm{r}}(\mathrm{mer})$ & 1,0 & 1,0 & 1,7 & 1,5 \\
\hline$f_{\mathrm{r}}$ (calculée) & 0,84 & 0,83 & 1,2 & 1,77 \\
\hline
\end{tabular}

\begin{tabular}{|c|c|c|}
\cline { 2 - 3 } \multicolumn{1}{c|}{} & $\begin{array}{c}\text { Baie } \\
\text { d'Hudson }\end{array}$ & $\begin{array}{c}\text { Mer de } \\
\text { Baffin }\end{array}$ \\
\hline$f_{\mathrm{r}}($ mer $)$ & 1,2 & 1,0 \\
\hline$f_{\mathrm{r}}$ (calculée) & 0,65 & 0,85 \\
\hline
\end{tabular}

OCEAN ATLANTIQUE

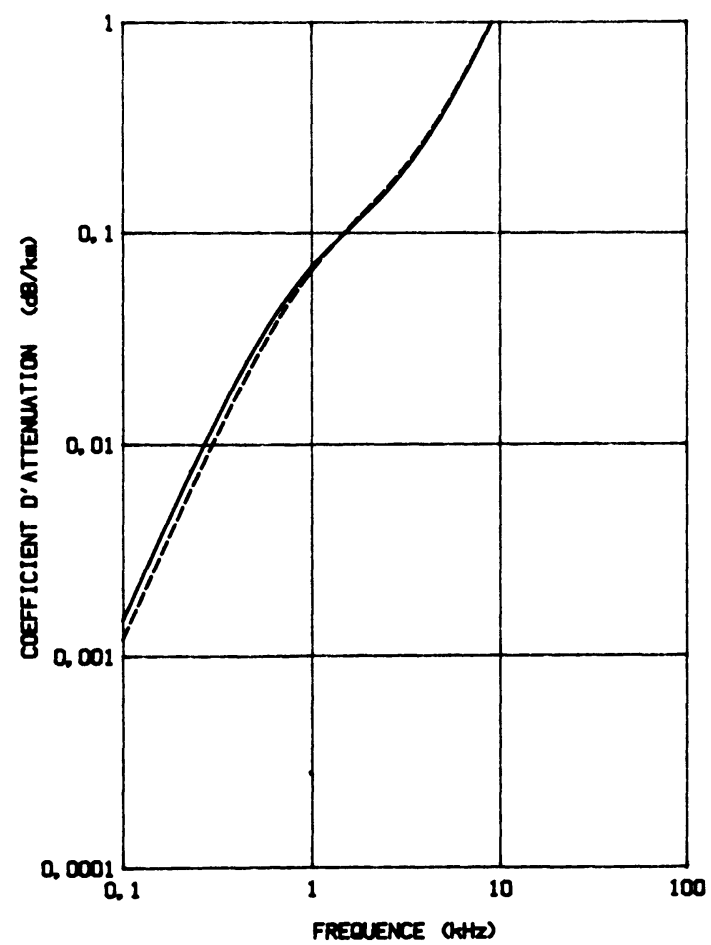

Fig. 3. - Atténuation acoustique de l'Océan Atlantique : $(\longrightarrow$ ) calculée d'après le modèle 12 , (-----) expérimentale.

[Sound attenuation in Atlantic Ocean. The full line represents the curve calculated from the diagram 12. The dotted line represents the experimental data.] 


\section{OCEAN PACIFIQUE}

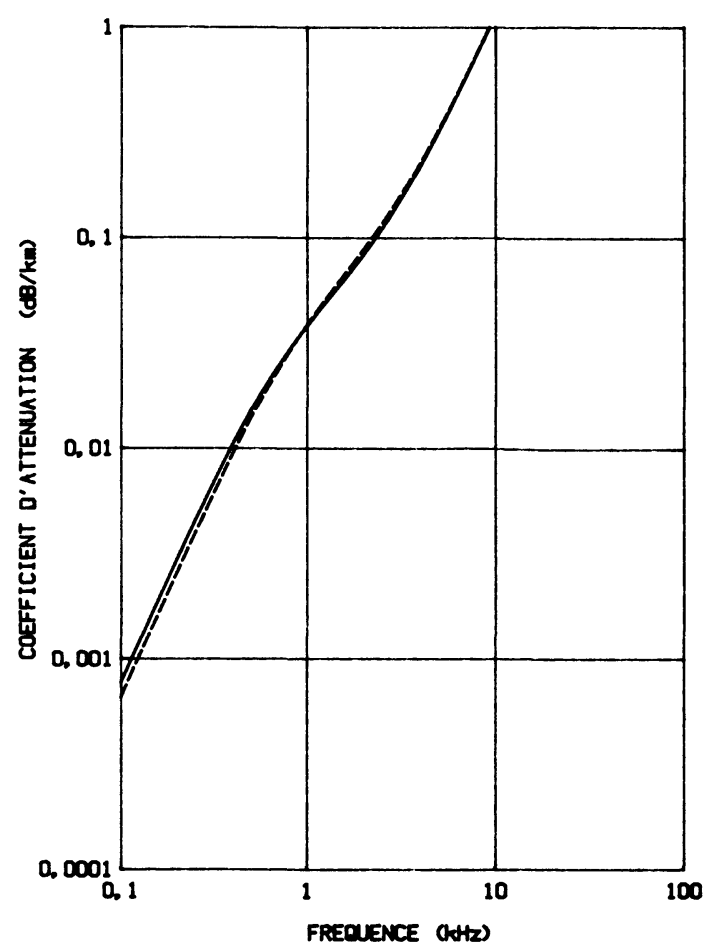

Fig. 4. - Atténuation acoustique de l'Océan Pacifique : $(\longrightarrow)$ calculée d'après le modèle 12 , (-----) expérimentale.

[Sound attenuation in Pacific Ocean. The full line represents the curve calculated from the diagram 12. The dotted line represents the experimental data.]

sulfate de magnésium. Par contre, l'atténuation dite " classique » qui tient compte des pertes par frottement visqueux et conductibilité thermique a été négligée. Sur ces mêmes figures, nous avons représenté en pointillés l'atténuation obtenue à partir des mesures de propagation acoustique à longue portée en océan.

\section{Discussion.}

L'examen du tableau I et des figures 3 à 5 montre que dans l'ensemble et en particulier pour les océans Atlantique et Pacifique, l'accord est satisfaisant, compte tenu des remarques suivantes :

- les expériences en mer sont très délicates à réaliser [18] et leurs résultats ne peuvent pas être très précis,

- pour faire le calcul de l'atténuation à partir de notre modèle, nous avons dû interpoler, voire même estimer parfois des grandeurs thermodynamiques, telles certaines constantes d'équilibre et certains volumes molaires partiels. Ces valeurs ne sont pas connues pour toutes les températures en milieu marin;

- tous nos calculs ont été effectués pour une pres-
MER MEDITERRANEE

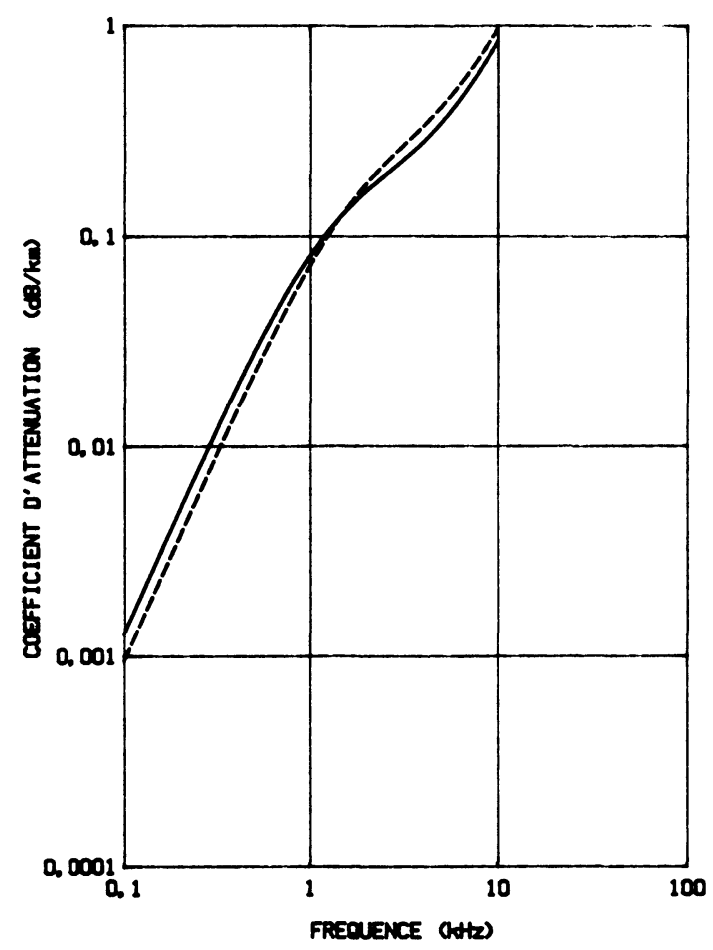

Fig. 5. - Atténuation acoustique de la Mer Méditerranée : (-) calculée d'après le modèle 12 , (-----) expérimentale.

[Sound attenuation in Mediterranean Sea. The full line represents the curve calculated from the diagram 12 . The dotted line represents the experimental data.]

sion de $1 \mathrm{~atm}$. Ceci introduit donc une approximation supplémentaire car les expériences en océan ont été réalisées en profondeur. Il est certain que la pression a pour effet de déplacer les constantes thermodynamiques et cinétiques,

- il n'a pas été tenu compte de la présence de phosphates et d'ammoniaque. Dans un récent travail, nous avons montré que ces composés pouvaient affecter la fréquence de relaxation [20]. L'Atlantique n'en contient que des quantités négligeables, mais ce n'est pas forcément le cas pour d'autres mers pour lesquelles nous ne disposons pas de données.

$\mathrm{Au}$ vu de l'ensemble de nos résultats, il semble que le modèle proposé permette de prévoir les caractéristiques d'expériences de propagation acoustique à longue portée si les conditions océanographiques et physicochimiques sont bien précisées.

\section{Remerciements.}

Les auteurs remercient Dr. R. H. Mellen pour des discussions très fructueuses. Ce travail a été réalisé avec l'aide de la D.R.E.T. Contrat no 82-34-025. 


\section{Bibliographie}

[1] Leonard, R. N., Combs, P. C. et Skidmore, L. R., J. Acoust. Soc. Am. 20 (1948) 868-873.

[2] Kurtze, G. et Tamm, K., Acustica 3 (1953) 33-48.

[3] Wilson, G. B. et Leonard, R., J. Acoust. Soc. Am. 26 (1954) 223-226.

[4] TAmm, K., Kurtze, G. et Kaiser, R., Acustica 4 (1954) 380.

[5] Tamm, K. et Haddenhorst, H., Acustica 4 (1954) 653.

[6] THORP, W. H., J. Acoust. Soc. Am. 38 (1965) 648-654.

[7] ThORP, W. H., J. Acoust. Soc. Am. 42 (1967) 270.

[8] Skretting, A. et Leroy, C. C., J. Acoust. Soc. Am. 49 (1971) 276-281.

[9] Yaeger, E., Fisher, F. H., Miceli, J. et Bressel, R., J. Acoust. Soc. Am. 53 (1973) 1705-1707.

[10] Simmons, V. P., Ph. D. Thesis, Department of Oceanography, University of California, San Diego (Ca) 1975.

[11] Mellen, R. H., Simmons, V. P. et Browning, D. G., J. Acoust. Soc. Am. 68 (1980) $248 ; 69$ (1981) 1660 ; 70 (1981) $143 ; 74$ (1983) 143.
[12] Waton, G., Mallo, P., Candau, S. J., J. Phys. Chem. 88 (1984) 3301.

[13] Mallo, P., Waton, G., Candau, S. J., Nouv. J. Chimie 8 (1984) 373.

[14] Waton, G., Candau, S., Rev. Cethedec 75 (1983) 50.

[15] Mellen, R. H., Browning, D. G., Simmons, V. P., J. Acoust. Soc. Am. 74 (1983) 143.

[16] Word, G. K. et Millero, F. J., Geochem. Cosmochim. Acta 36 (1972) 92.

[17] Millero, F. J., Geochem. Cosmochim. Acta 45 (1982) 11.

[18] Mellen, R. H., Browning, D. G., Simmons, V. P., J. Acoust. Soc. Am. 70 (1981) 143.

[19] Waton, G. et Candau, S., Rev. Cethedec 67 (1981) 83.

[20] Mallo, P., Waton, G., Mellen, R. H., Nouv. J. Chimie, soumis pour publication. 